\title{
Radiomètre en ondes millimétriques pour l'étude de l'atmosphère. Utilisation d'éléments quasi-optiques
}

\author{
C. Prigent, P. Abba, G. Beaudin, M. Gheudin et G. Ruffié \\ Département de Radioastronomie Millimétrique, Observatoire de Paris, Meudon, France
}

(Reçu le 13 octobre 1987, accepté le 30 novembre 1987)

\begin{abstract}
Résumé. - Nous présentons un système radiométrique en ondes millimétriques pour l'étude des raies atmosphériques de l'oxygène et de la vapeur d'eau situées entre 100 et $200 \mathrm{GHz}$. Après une description du principe du radiosondage, les divers éléments du récepteur sont exposés. L'accent est porté sur les tehniques quasi-optiques utilisées : filtre, interféromètre, rotateur de polarisation.
\end{abstract}

\begin{abstract}
We present a millimeter-wave radiometric system to study oxygen and water vapor atmospheric lines between 100 and $200 \mathrm{GHz}$. After a description of the remote sensing principle, we describe some elements of the receiver. The quasi-optical part of the receiver is emphasized: filter, interferometer, polarization rotator.
\end{abstract}

\section{Introduction.}

Depuis les années 1970, à bord de satellites, le sondage passif de l'atmosphère en infra-rouge a fourni aux météorologues des données de base pour leurs modèles de prévisions. Mais en cas de couverture nuageuse, le sondage infra-rouge est impuissant et la contribution de la radiométrie micro-ondes s'est récemment révélée fructueuse.

Les satellites américains NOAA/TIROS N (MSU, AMSU-A et B) ont montré la voie en prouvant que l'on pouvait extraire, à partir des mesures radiométriques, les deux paramètres physiques essentiels de l'atmosphère - le gradient de température et la distribution en vapeur d'eau - dans toutes les directions de l'espace.

\section{Extraction des paramètres météorologiques.}

Le spectre de l'atmosphère dans le domaine millimétrique est dominé par les raies d'absorption de la vapeur d'eau et de l'oxygène.

Jusqu'à environ $40 \mathrm{~km}$, dans le domaine des fréquences millimétriques, le facteur essentiel d'élargissement des raies est la pression. En conséquence, la distribution en altitude d'un constituant de

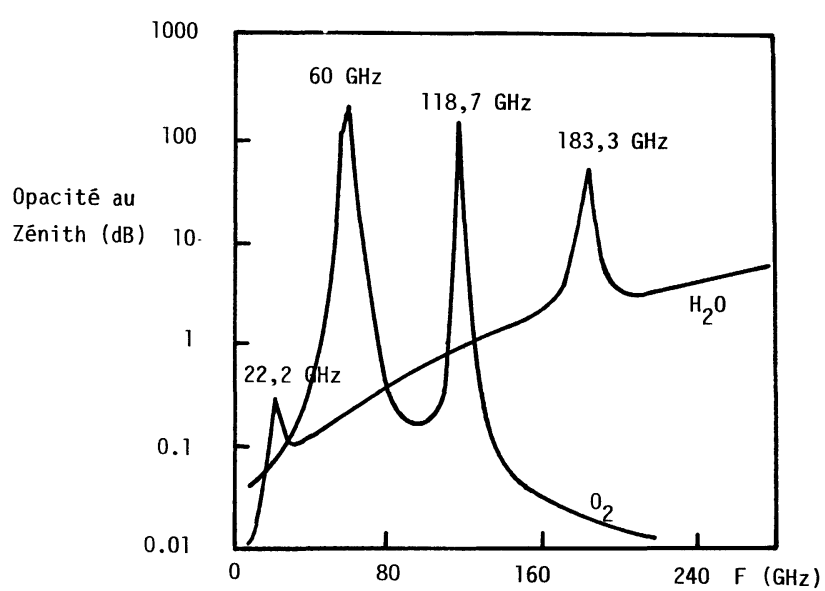

Fig. 1. - Atténuation atmosphérique (au niveau de la mer pour une atmosphère standard U.S.).

[Atmospheric attenuation (at sea level for a U.S. standard atmosphere).]

l'atmosphère peut se déduire de la mesure de l'élargissement d'une de ses raies d'absorption. Réciproquement, si la distribution verticale d'un constituant est connue, cette mesure nous donnera le profil en température de l'atmosphère [1]. 
Plus précisément, écrivons l'équation de base du transfert radiatif qui relie la température de brillance aux paramètres atmosphériques. En négligeant les phénomènes de diffusion :

$$
\begin{aligned}
& T_{\mathrm{B}}(f)=\underbrace{\varepsilon_{\mathrm{s}} T_{\mathrm{s}} \mathrm{e}^{-\int_{0}^{\infty} \alpha(f, z) \mathrm{d} z}}_{\text {émission de la sur- }}+\underbrace{\int_{0}^{\infty} \alpha(f, z) \mathrm{e}^{-\int_{z}^{\infty} \alpha\left(f, z^{\prime}\right) \mathrm{d} z^{\prime}} T(z) \mathrm{d} z}_{\text {émission de chaque couche de }} \\
& \text { face terrestre, atté- l'atmosphère, chacune étant atténuée } \\
& \text { nuée par l'atmos- par l'atmosphère au-dessus d'elle } \\
& \text { phère }
\end{aligned}
$$$$
+\underbrace{\left(1-\varepsilon_{\mathrm{s}}\right) \mathrm{e}^{-\int_{0}^{\infty} \alpha(f, z) \mathrm{d} z} \int_{0}^{\infty} \alpha(f, z) \mathrm{e}^{-\int_{0}^{z} \alpha\left(f, z^{\prime}\right) \mathrm{d} z^{\prime}} T(z) \mathrm{d} z}
$$$$
\text { radiations émises vers le sol et réfléchies par la surface }
$$
terrestre

$T_{\mathrm{B}}(f) \quad=$ température de brillance à la fréquence $f$

$T(z)=$ température de l'atmosphère à l'altitude $z$

$\varepsilon_{\mathrm{s}} \quad=$ émissivité de la surface terrestre

$T_{\mathrm{s}} \quad=$ température de la surface terrestre

$\alpha(f, z)=$ coefficient d'absorption de l'atmosphère à la fréquence $f$ à l'altitude $z$.

En mesurant la température de brillance dans une partie du spectre où l'absorption de l'oxygène domine, $\alpha(f, z) \simeq \alpha_{\mathrm{O}_{2}}(f, z)$. La concentration de l'oxygène est constante et connue dans l'atmosphère jusqu'à $80 \mathrm{~km}$. Donc, $\alpha(f, z)$ étant connu, des mesures de $\varepsilon_{\mathrm{s}}, T_{\mathrm{s}}$ et $T_{\mathrm{B}}(f)$, on déduit le profil en température $T(z)$.

Le profil en vapeur d'eau de l'atmosphère est obtenu par le sondage dans des régions spectrales où l'absorption due à la vapeur d'eau domine: $\alpha(f, z) \simeq \alpha_{\mathrm{H}_{2} \mathrm{O}}(f, z) . \varepsilon_{\mathrm{s}}, T_{\mathrm{s}}$ et $T(z)$ sont supposés connus et la concentration en vapeur d'eau, directement proportionnelle à $\alpha_{\mathrm{H}_{2} \mathrm{O}}(f, z)$, est modifiée dans $\alpha(f, z)$ jusqu'à ce que les valeurs calculées et mesurées de $T_{\mathrm{B}}(f)$ coïncident. Dans les canaux les plus transparents (fenêtres atmosphériques), le premier terme de l'équation (1) domine et à partir des mesures de $T_{\mathrm{B}}(f)$ on peut approcher l'émissivité et la température de surface de la Terre.

Comment choisir les fréquences de sondage pour obtenir la résolution en altitude des profils ?

En supposant la surface de la Terre peu réfléchissante, récrivons l'équation (1) :

$$
T_{\mathrm{B}}(f)=\varepsilon_{\mathrm{s}} T_{\mathrm{s}} \mathrm{e}^{-\tau(f)}+\int_{0}^{\infty} W(f, z) T(z) \mathrm{d} z
$$

$\tau(f)=\int_{0}^{\infty} \alpha(f, z) \mathrm{d} z$ est l'opacité au zénith.

$W(f, z)$ est appelée fonction de poids. Elle mesure la contribution à la température de brillance, de la tranche $\mathrm{d} z$ à l'altitude $z$.

$$
W(f, z)=\alpha(f, z) \mathrm{e}^{-\int_{z}^{\infty} \alpha(f, z) \mathrm{d} z}
$$

La fonction de poids $W(f, z)$ admet généralement un maximum à une altitude $z_{\max }$ qui varie avec la fréquence. En sondant à la fréquence dont la fonction de poids admet $z_{i}$ pour maximum, on obtient préférentiellement des informations sur l'atmosphère à l'altitude $z_{i}$.

Le choix des canaux de sondage est donc dicté par l'étude des fonctions de poids.

Les $z_{i(\max )}$ (maximum de fonction de poids) doivent couvrir la gamme d'altitude à étudier.

A l'aide de canaux très étroits par rapport à la largeur de la raie, on mesure l'absorption à différentes fréquences autour de la fréquence centrale de la raie. Les raies sont symétriques en première approximation. On mesure les températures de brillance avec un récepteur hétérodyne dont l'oscillateur local est accordé sur la fréquence centrale de la raie. Grâce à la symétrie, fréquence image et fréquence signal contiennent la même information, c'est-à-dire correspondent à la même altitude $z_{i}$ sondée.

La résolution verticale des profils atmosphériques est directement liée à la résolution spectrale du sondeur, c'est-à-dire la largeur de bande des canaux du sondeur. Il y a donc un compromis entre résolution verticale et sensibilité (qui est inversement proportionnelle à la racine de la largeur de bande). La sensibilité doit être meilleure que le Kelvin dans tous les canaux. 


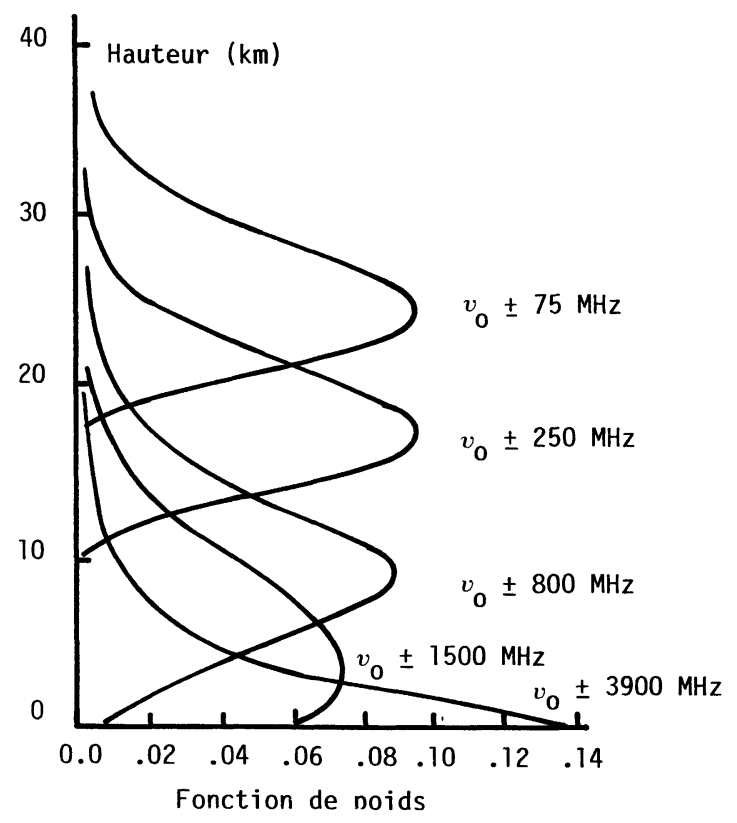

a)

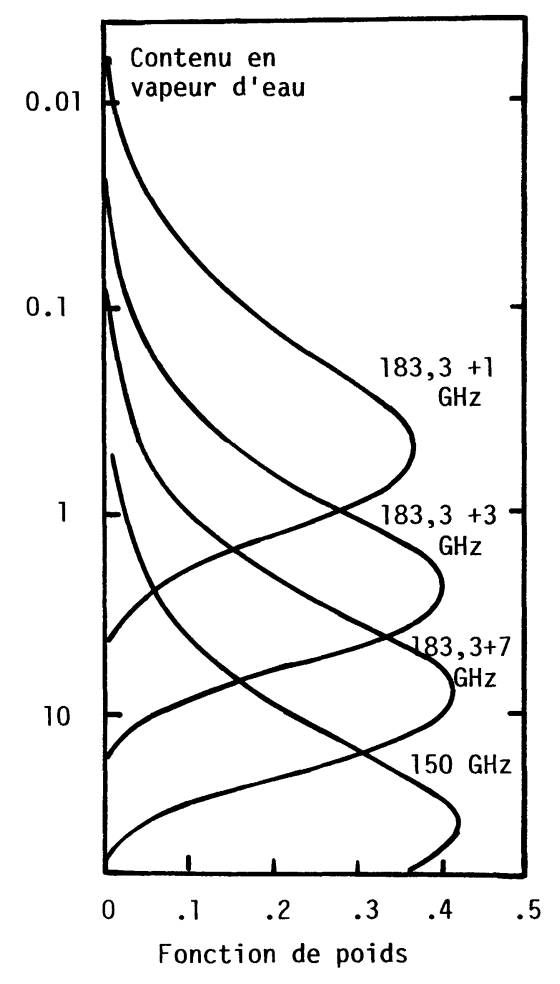

b)

Fig. 2. - (a) Fonctions de poids de $\mathrm{O}_{2}$ autour de $118,75 \mathrm{GHz}$; (b) fonctions de poids pour la vapeur d'eau autour de $183,3 \mathrm{GHz}$.

[(a) Weighting functions for $\mathrm{O}_{2}$ around $118.75 \mathrm{GHz}$; (b) weighting functions for $\mathrm{H}_{2} \mathrm{O}$ around $183.3 \mathrm{GHz}$.]

Pour répondre aux exigences des météorologues, la répartition suivante des canaux est proposée :

- cinq canaux autour de la raie $118 \mathrm{GHz}$ de l'oxygène donnent un profil en température de l'atmosphère entre 0 et $25 \mathrm{~km}$;

- pour la détermination du profil vertical de vapeur d'eau, trois canaux situés autour de la raie à $183 \mathrm{GHz}$ de l'eau analysent la tranche de $2-3 \mathrm{~km}$ à 8$10 \mathrm{~km}$;

— de plus, deux canaux «fenêtre » à $110 \mathrm{GHz}$ et $150 \mathrm{GHz}$ corrigent des émissions du sol, les mesures à $118 \mathrm{GHz}$ et $183 \mathrm{GHz}$.

\section{Synoptique du sondeur atmosphérique en ondes millimétriques.}

L'émission atmosphérique est collectée par une antenne parabolique. Le signal large bande qu'elle délivre sous forme d'un faisceau gaussien est séparé en quatre bandes par un filtrage quasi-optique. Ces diverses bandes de fréquences $\left(« \mathrm{O}_{2} », \ll \mathrm{H}_{2} \mathrm{O} »\right.$, et deux fenêtres) sont envoyées à des mélangeurs hétérodynes à diode Schottky GaAs faible bruit. Les bandes sont analysées plus finement par les canaux des radiomètres qui présentent des résolutions différentes selon la partie du spectre observée.

L'étalonnage des récepteurs est effectué par commutation du faisceau sur une charge froide à $77 \mathrm{~K}$ (ou le fond du ciel à $3 \mathrm{~K}$ ) et sur une charge chaude à $300 \mathrm{~K}$.

Les données recueillies sont transmises au sol pour être dépouillées (éventuellement en temps réel). Les composants millimétriques actifs et passifs (mélangeurs, multiplicateurs, amplificateurs) sont dérivés de ceux utilisés par les radioastronomes observant des raies moléculaires dans le domaine des ondes millimétriques. Ils sont décrits dans des communications antérieures [2].

\section{Filtrage et injection quasi-optique du signal.}

Au-delà de $100 \mathrm{GHz}$, l'importance des pertes dans les guides nous conduisent à préférer un mode de propagation en espace libre. Mais contrairement à l'optique classique, la dimension des dispositifs (filtres, lentilles...) n'est pas très grande devant la longueur d'onde et impose de prendre en compte les phénomènes de diffraction. Dans un plan perpendiculaire à la direction de propagation, la répartition en énergie n'est plus constante mais d'allure gaussienne [3].

3.1 CORNETS ET ÉlÉMENTS FOCALISANTS. - A l'entrée des mélangeurs, les faisceaux sont recueillis par des cornets. Des lentilles de diélectrique [4] ou des miroirs ellipsoïdaux sont utilisés pour concentrer l'énergie sur ces cornets et pour limiter la dimension des faisceaux dans l'ensemble du récepteur. 
charge froide $(77 \mathrm{~K})$

ou ciel ( $3 \mathrm{k})$

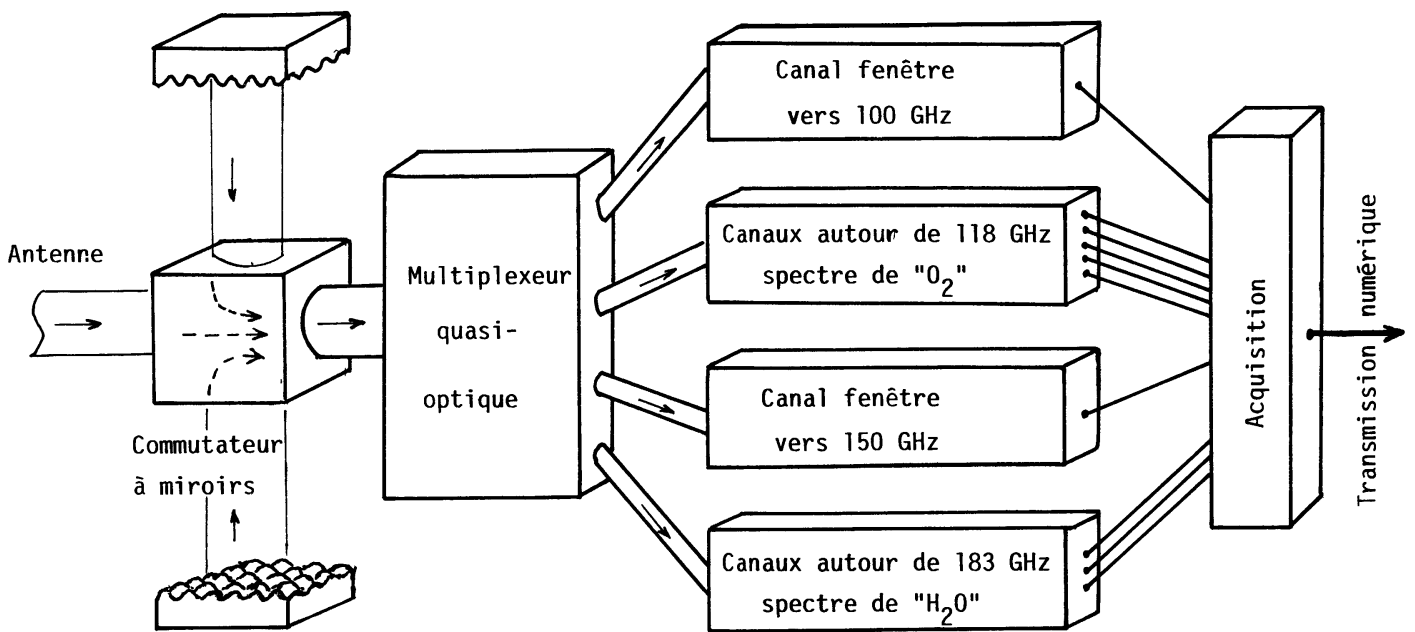

Source chaude

$(\sim 300 \mathrm{~K})$

Fig. 3. - Schéma du récepteur.

[Receiver bloc.]

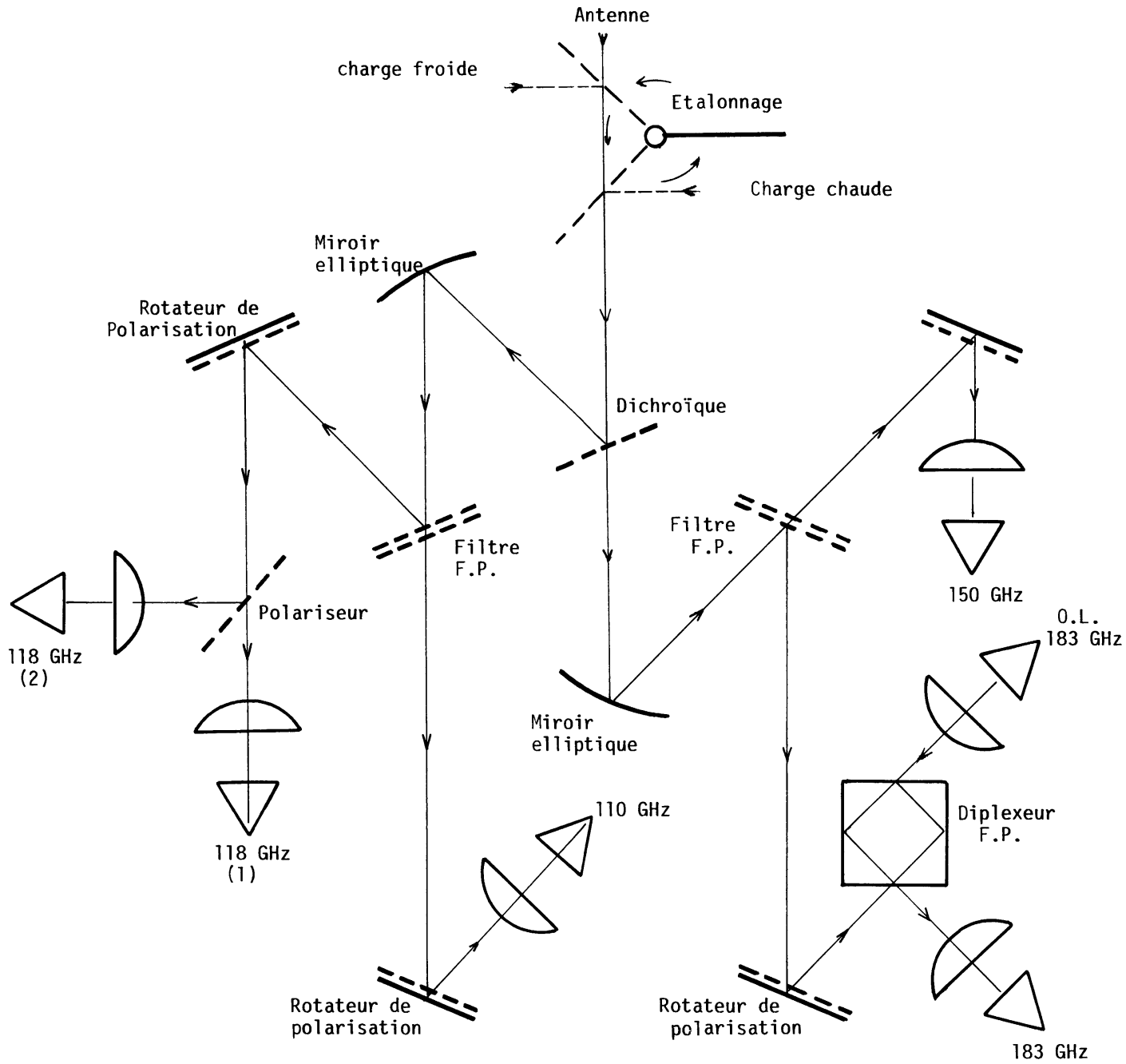

Fig. 4. - Schéma de la partie quasi-optique.

[Quasi-optical part of the receiver.] 


\subsection{Filtrage des CANAUX.}

3.2.1 Lame dichröque. - Les bandes de fréquences les plus éloignées sont séparées par une lame dichroïque. Celle-ci est constituée par une feuille métallique perforée de trous circulaires. Chaque trou se comporte comme un guide dont le diamètre détermine la fréquence de coupure $\left(\lambda_{\mathrm{c}}=3,412 \mathrm{~d} / 2\right.$, longueur d'onde de coupure d'un guide circulaire pour le mode fondamental). Les fréquences inférieures à la fréquence de coupure sont réfléchies. Les pertes dans la bande transmise dépendent de la fréquence (Fig. 5).

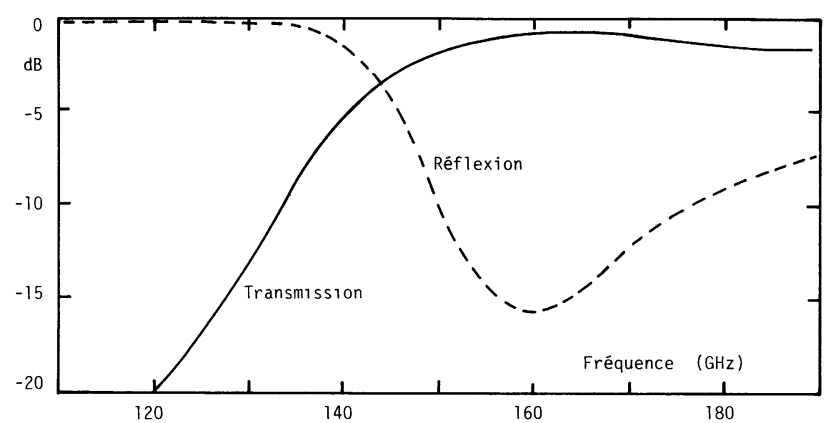

Fig. 5. - Coefficients de réflexion et de transmission de la lame dichroïque.

[Reflection and transmission coefficients of the dichroic plate.]

L'épaisseur $e$ de la lame contrôle la raideur du filtre. Lorsque $e$ croît, la raideur du filtre augmente mais la bande passante rétrécit. En pratique $e$ est voisin de $\lambda_{\mathrm{g} 0} / 2$ ( $\lambda_{\mathrm{g} 0}$ : longueur d'onde guidée dans les trous au centre de la bande passante). D'autre part, la lame dichroïque constitue un réseau plan de trous rayonnants. La formation de lobes de réseau est évitée si le pas du réseau $p$ et l'angle d'incidence $\theta_{\mathrm{i}}$ vérifient :

$$
p<\lambda_{\min } /\left(1+\sin \theta_{\mathrm{i}}\right)
$$

( $\lambda_{\min }:$ longueur d'onde minimale à transmettre).

3.2.2 Interféromètre Fabry-Pérot. - Utilisé pour séparer deux bandes proches (bande à $118 \mathrm{GHz}$ et canal fenêtre vers $110 \mathrm{GHz}$ ), il est constitué de deux surfaces parallèles partiellement réfléchissantes.

L'analyse classique [5] des réflexions multiples entre les deux surfaces permet d'écrire le facteur de transmission en puissance :

$$
|\tau|^{2}=\frac{\xi T^{2}}{(1-\xi R)^{2}+4 \xi R \sin ^{2}\left(\frac{\Delta \phi}{2}-\phi_{\mathrm{r}}\right)}
$$

avec :

$\left|\sqrt{R} \mathrm{e}^{i \phi_{\mathrm{r}}}\right|^{2}:$ coefficient de réflexion en puissance d'une surface
$T$ et $A \quad$ : coefficients de transmission et d'absorption en puissance d'une surface $(T=1-R-A)$

$\xi$

: coefficient de transmission en puissance sur un aller entre les 2 surfaces.

$$
\Delta \phi=\frac{4 \pi d}{\lambda} \sqrt{n^{2}-\sin ^{2} \theta_{i}}
$$

$d$ est choisi en fonction de $n, \theta_{\mathrm{i}}$ et des longueurs d'onde $\lambda$ à transmettre. L'allure de la courbe de réponse du filtre est donnée à la figure 7 .

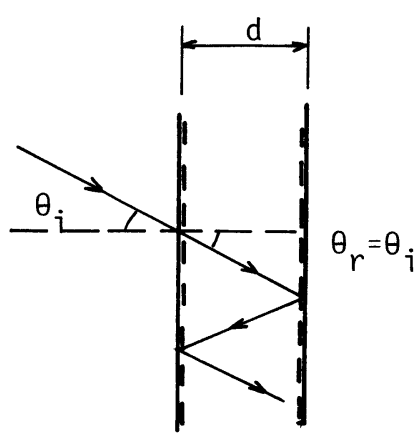

a)

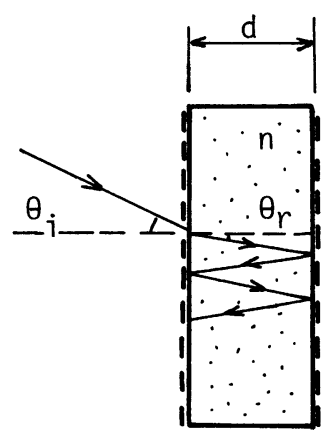

b)
Fig. 6. - (a) Lames métallisées ; (b) Lame de diélectrique métallisée sur les 2 faces.

[(a) Metallized plates ; (b) Dielectric plate metallized on both surfaces.]

Les pics de transmission sont d'autant plus pointus que $R$ est proche de 1 . Les surfaces semi-réfléchissantes sont constituées par des lames de diélectrique (quartz, mylar...), sur lesquelles on imprime un réseau métallique; différents types de réseaux ont été étudiés : bandes croisées, pavés, motifs en croix de Jérusalem, ... [6].

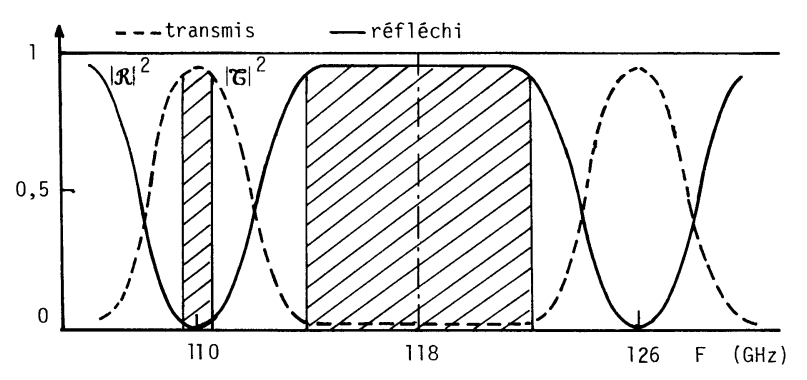

Fig. 7. - Coefficients de réflexion et de transmission de l'interféromètre Fabry-Perot.

[Reflection and transmission coefficients of the FabryPerot interferometer.]

3.3 RotATEUR DE POLARISATION. - Le rayonnement émis par l'oxygène et la vapeur d'eau dans l'atmosphère n'est pas polarisé. Mais les canaux 
sensibles aux radiations du sol, c'est-à-dire les canaux fenêtres à 110 et $150 \mathrm{GHz}$ et les canaux situés dans les ailes de raies, reçoivent des radiations réfléchies par le sol, la mer, les nuages. Or, par réflexion, le rayonnement se polarise. Les canaux qui voient le sol sont donc bruités par un rayonnement polarisé. La direction de polarisation par rapport au satellite n'est pas figée : non seulement elle est sensible au relief de la surface qui réfléchit l'onde (tout particulièrement à la surface de la mer), mais bien sûr varie avec l'angle sous lequel le satellite balaye la zone étudiée.

Nous envisageons donc la possibilité d'analyser successivement deux composantes perpendiculaires du rayonnement reçu. Les mélangeurs de réception utilisés n'étant sensibles qu'à une seule polarisation linéaire, nous avons réalisé et testé au laboratoire un rotateur de polarisation se plaçant en amont de chaque récepteur. Ce rotateur évite de doubler le nombre de radiomètres ou de faire subir une rotation de $90^{\circ}$ au mélangeur et aux éléments qui lui sont liés (oscillateur local, préampli FI).

Le système est constitué par une nappe de fils conducteurs parallèles, placée à une distance d'un quart de longueur d'onde d'un miroir plan. L'appareil travaille en réflexion. Si la période $p$ du réseau de fils est très inférieure à la longueur d'onde, le coefficient de réflexion $R$ de celui-ci pour une polarisation parallèle aux fils est proche de 1 , et est pratiquement nul pour une polarisation orthogonale [7].

Dans un premier temps, les fils de la grille sont orientés parallèlement ou perpendiculairement à la direction privilégiée par le mélangeur. Supposons celle-ci verticale : c'est alors la composante verticale du rayonnement incident qui est détectée par le récepteur.

Puis la grille est tournée de $45^{\circ}$ dans son plan (Fig. 8) : c'est maintenant la composante horizontale du rayonnement incident qui est reçue par le mélangeur car elle ressort verticalement du dispositif.

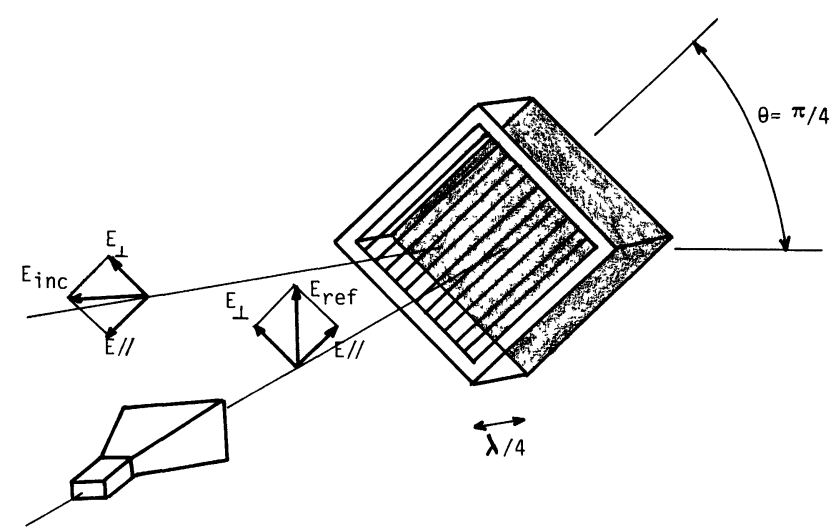

Fig. 8. - Rotateur de polarisation.

[Polarization rotator.]

Plaçons-nous dans le cas où $E_{\text {inci }} \perp E_{\text {reçu }}$ (le cas $E_{\text {inci }} / / E_{\text {reçu }}$ est immédiat) :

pour une grille parfaite $(R=1)$, le rapport puissance reçue/puissance émise est égal à $\sin ^{2}$ $\left[(\pi / 2)\left(f / f_{0}\right)\right]\left(f_{0}\right.$ fréquence centrale de la bande à analyser) : le taux de polarisation croisée reçue est

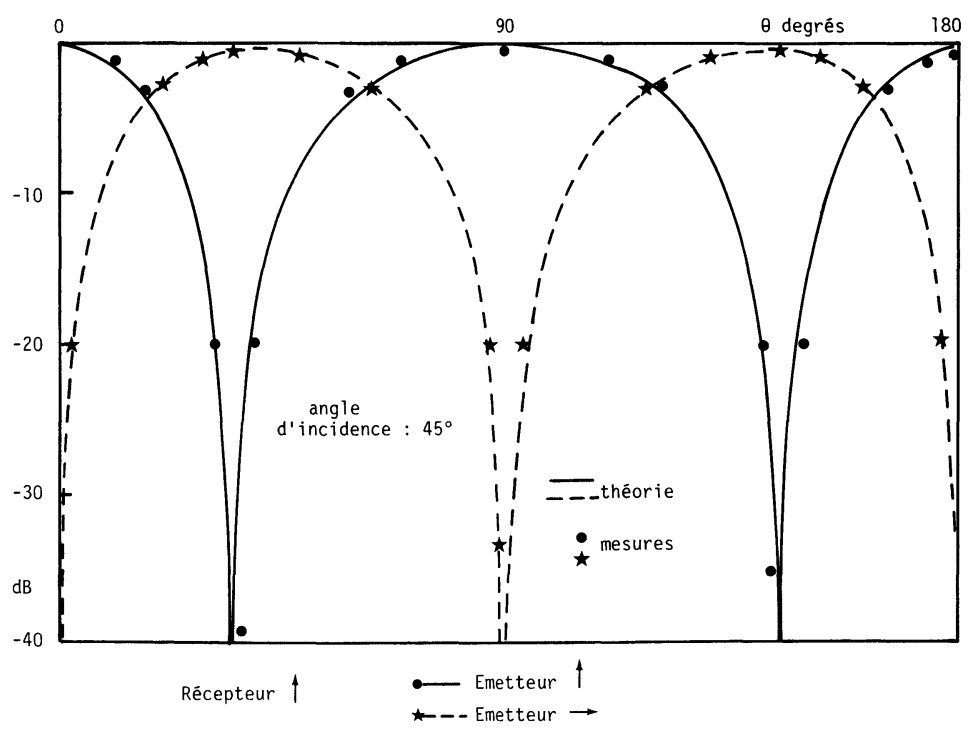

Fig. 9. - Transmission du rotateur de polarisation.

[Transmission of the polarization rotator.] 
donc inférieur à $1 \%$ en puissance sur une bande de $12 \%$.

Si la grille n'est pas parfaite $(R \simeq 1)$, on peut montrer que la distance optimale entre le miroir et les fils est légèrement supérieure à $\lambda_{0} / 4$.

Pour une plus grande facilité de réalisation, la distance grille-miroir peut être choisie égale à $3 \lambda_{0} / 4,5 \lambda_{0} / 4 \ldots$, mais au détriment de la bande passante.

La grille n'est utilisable que pour un angle d'incidence $i$ non nul. Une simple considération géométrique montre alors que $\theta$ est en fait donné par: $\tan \theta=\cos i$.

Ce système présente de faibles pertes d'insertion (Fig. 9). Il est en outre très simple à mettre en œuvre.

\section{Conclusion.}

Notre étude a démontré la faisabilité d'un système radiométrique atmosphérique dans la bande 100$200 \mathrm{GHz}$.

L'expérience acquise depuis de nombreuses années par notre laboratoire dans le développement de systèmes de réception à faible bruit (pour la radioastronomie et pour l'étude de composants mineurs de l'atmosphère), ainsi que les tests favorables obtenus récemment sur les dispositifs quasioptiques, nous encouragent à poursuivre la réalisation d'un instrument spécialisé pour l'étude de l'atmosphère. Celui-ci pourrait être directement appliqué à la future génération de sondeurs météorologiques embarqués sur satellites, tel METEOSAT seconde génération [8].

\section{Bibliographie}

[1] Scott, N. A., Chedin, A., Wahiche, C., Progrès récents en télédétection tridimensionnelle de paramètres atmosphériques, Météorologie (juin, août 1985).

[2] Beaudin, G., Gheudin, M., Composants millimétriques, cryogéniques et à faible bruit, IV ${ }^{\text {es }}$ J.N.M. (Lannion) juin 1984, p. 104.

[3] Goldsmith, P. F., Quasi-optical techniques at millimeter and submillimeter wavelengths, IR and MM waves, Ed. K. J. Button 6 (1982).

[4] Gheudin, M., Beaudin, G., Récepteurs millimétriques à faible bruit, $\mathrm{IV}^{\mathrm{es}}$ J.N.M. (Lannion) juin 1984, p. 262.
[5] Born, M., Wolf, E., Principles of optics (Pergamon Press) 1975.

[6] Arnaud, J. A., Pelow, F. A., Resonant-grid quasioptical diplexers, Bell Syst. Tech. J. 54, $\mathrm{n}^{\circ} 2$ (1975).

[7] Marcuvitz, N., Waveguide Handbook 5 (McGrawHill) 1951.

[8] Chedin, A., Pick, D., Rizzi, R., Meteosat 2nd generation, consultant report, ESA, March 1985. 